\title{
A Survey on Vertical Handoff Decision Making in Next Generation Wireless Heterogeneous Networks
}

\author{
Sudhir L. Mane ${ }^{1}$, Ragib Nasri ${ }^{2}$ \\ Department of Computer Engineering, Savitribai Phule Pune University, Pune, SKNCOE, Vadgoan, Pune, India \\ Professor, Faculty of Computer Engineering, Savitribai Phule, Pune, University, Pune, SKNCOE, Vadgoan, Pune, India.
}

\begin{abstract}
Heterogeneous wireless network will be dominant in next generation wireless networks (NGWN). Increasing user demands and the growing interest of service providers to offer diverse applications have motivated the integration of heterogeneous wireless networks which enable the users to move seamlessly across different types of networks and to enjoy anytime, anywhere services. The objective is to determine the conditions under which handoff should be performed. Nowadays mobile handsets are capable of having different network interfaces. There are multiple criteria for handoff in NGWN. Handoff decision algorithm decides the optimal target network by considering a set of decision parameters including available bandwidth, velocity, throughput, monetary cost, security and user preference. One of the technical challenges with this integration and interfacing of heterogeneous wireless networks is handoff decision that is used to choose the optimal network depending upon the type and service requirements demanded by the user's application
\end{abstract}

Keywords: Mobility management, Next generation wireless networks, Media independent handoff, Vertical handoff management, Handoff types.

\section{Introduction}

It consists of various wireless networks (e.g. Worldwide Interoperability for Microwave Access (WiMAX) and Wireless Fidelity (Wi-Fi)) and cellular communications (e.g. beyond the third generation $(\mathrm{B} 3 \mathrm{G})$ and the fourth generation (4G)). The adoption of wireless technologies is increasing at a very fast rate. This trend is basically due to factors such as:

a) The miniaturization of devices such as laptops, Personal Digital Assistants (PDA), and notetbook(tabs).

b) The multiple networking interfaces available in most devices.

c) The availability of several wireless technologies such as Wireless Fidelity (Wi-Fi), Worldwide interoperability for Microwave Access (WiMAX), and Universal Mobile Telecommunications System (UMTS).

\section{A. Mobility Management}

For efficient delivery of services to the mobile users, the next-generation wireless networks require mechanisms known as mobility management where the location of every user is proactively determined before the service is delivered. Tracking the location of the mobile subscribers, allowing continuity of calls and other services is the objective of mobility management. Mobility management is the combination of location management and handoff management. Mobility management is the essential technology that supports roaming users with mobile terminals to enjoy their services in progress through wireless networks.

Mobility management contains two distinct but related components: location management and handoff management. The operations of the two components are shown in Fig $1.1 \mathrm{a} \& \mathrm{~b}$ respectively.

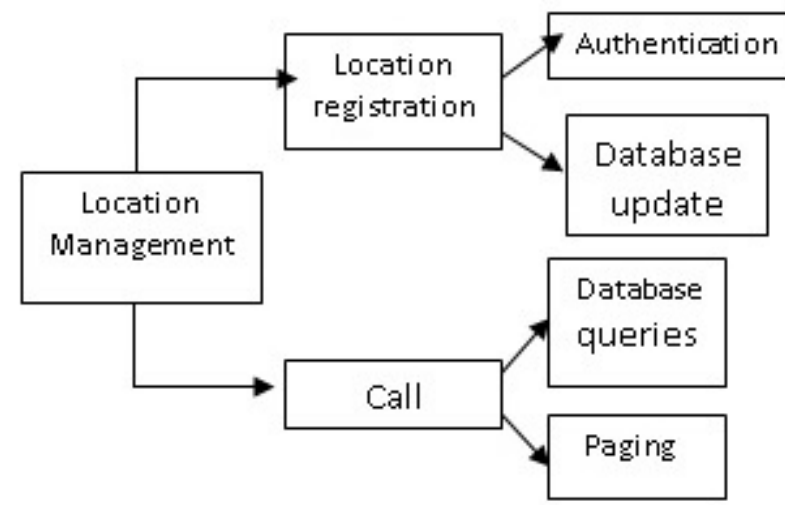

Figure 1.1 (a): Location Management

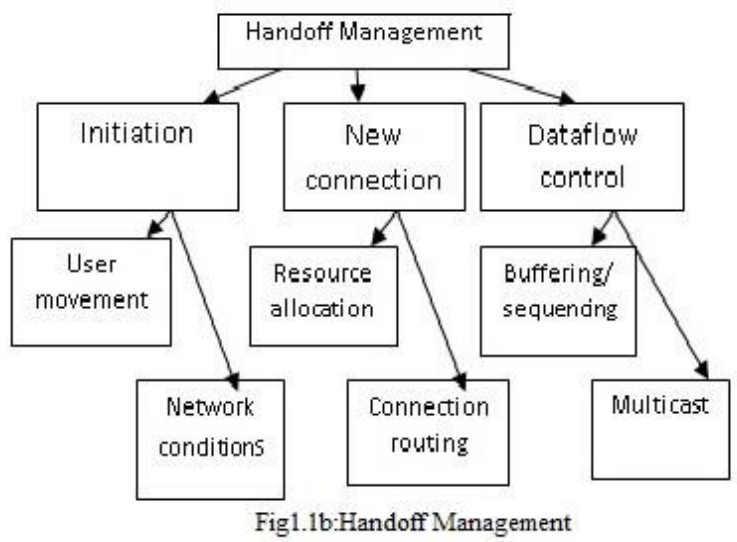

B. Types of Handoff

Handoffs are classified as two types with respect to the behavior of a mobile terminal for allowing itself for a new connection. They are hard handoffs and smooth handoffs. A handoff in which a mobile terminal releases its existing connection with a base station before allowing itself connect to a new base station is considered as hard handoff. A mobile 


\section{International Journal of Science and Research (IJSR) \\ ISSN (Online): 2319-7064}

Index Copernicus Value (2013): 6.14 | Impact Factor (2014): 5.611

terminal connecting itself with a new base station before releasing its already existing connection is treated as soft handoff. Handoffs are classified as four types with respect to „who controls the handoff decision". Fig 1.2 depicts classification of various types of handoffs. If the control agent for handoff decision resides on the network side then it is called as network controlled Handoff (NCHO), if it resides on the mobile terminal then it is called as mobile controlled handoff $(\mathrm{MCHO})$. If the mobile terminal assists the control agent, who is on the network side, in giving the primary information then it is called as mobile assisted handoff (MAHO). In case, if the network assists the control agent who is on the mobile terminal side then it is called as network assisted handoff (NAHO).

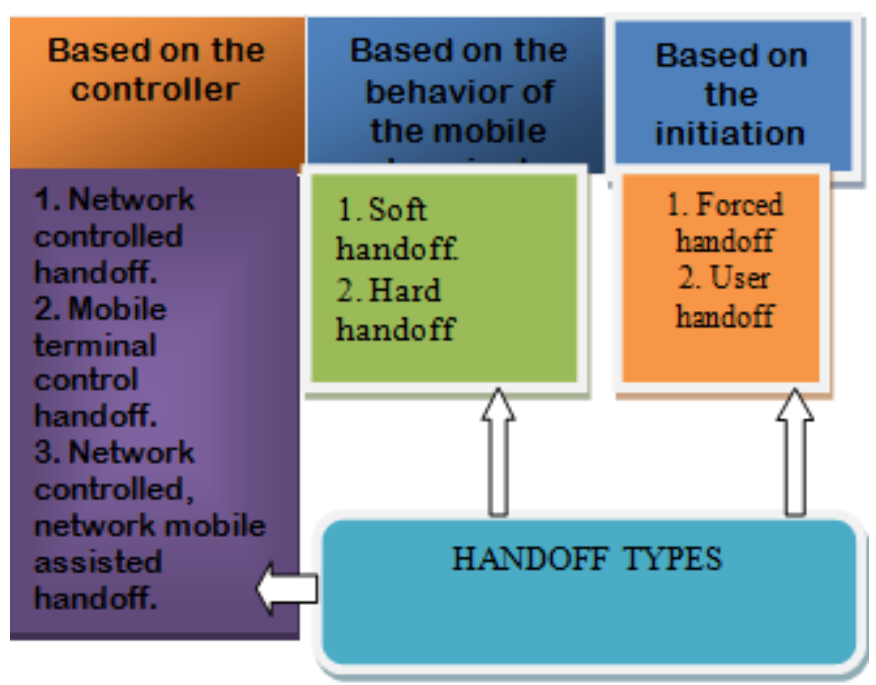

Figure 1.2: Classification of Handoff

\section{Motivation}

Next generation heterogeneous wireless systems will be expected to provide end-users with a usage model for wireless devices that will allow them to roam seamlessly among different networks, using multimodal wireless devices. But there should be minimum handoff overheads like delays, connection drops and packet losses, both in case of intra and inter-technology handoff scenario (vertical and horizontal handoffs). Next generation communications will offer a wide range of services available to users anywhere at any time. Users will have access to multiple networks at the same time. So there is a need that the user should be able to roam seamlessly among the multiple access network technologies for which handoffs are needed. Although there are many types of handoffs are available to precede the connection but due to the improper handoff decision, a handoff overhead like delays, connection drops and packet losses occurs. Handoff decision algorithms are essential components of the next generation heterogeneous wireless networks that are needed to satisfy the requirement of seamless roaming across the networks. These algorithms helps us gain a good Quality of Service (QoS)at a wide range of networks and help improving the efficiency by selecting the best network among different available networks by applying some parameters and criteria for selection. An efficient handoff decision algorithm that takes into account services requirements considers users preferences and guaranties seamless handover over heterogeneous technologies.

\section{Vertical Handoff Decision Making Parameters}

The decision for vertical handoff may depend on various parameters like Bandwidth, Received Signal Strength (RSS), signal to inference ratio (SIR), cost, latency, security, velocity,battery power, user preferences, service capacities and Quality of service (QoS). In order to design a Vertical Handoff (VHO) mechanism for next generation wireless networks, it is essential to study the existing VHO mechanisms. The study of existing mechanisms will assist in the identification of important parameters for VHO mechanism.

\section{A. Available Bandwidth}

Bandwidth is a measure of the width of a range of frequencies. It refers to the data rate supported by a network connection or interface. It measures how much data can be sent over a specific connection in a given amount of time. In order to provide seamless handoff for QoS in wireless environment, there is a need to manage bandwidth requirement of mobile node during movement. Bandwidth is generally known as the link capacity in a network. Higher offered bandwidth ensures lower call dropping and call blocking probabilities; hence higher throughput. Bandwidth handling should be an integral part of any of the handoff technique.

\section{B. Speed}

It is the speed at which the Mobile Terminal (MT) is moving. In vertical handoff algorithms, the speed factor has a large and important decisions binding effect than traditional handoff decision algorithms horizontal handoff. When the users travel at high speed within a network coverage area is discouraged the idea to initiate vertical handoff process because after a short period of time the user will have to go back to the initial network because it will get out from under cover network host.

\section{Received Signal Strength (RSS)}

RSS is the most widely used criterion because it is easy to measure and is directly related to the service quality. Majority of existing horizontal handover algorithms use RSS as the main decision criterion, and RSS is an important criterion for VHD algorithms as well, but it is not enough for a complete decision. It is the strength of the signal received, as the RSS of the neighboring network rises above the threshold the Vertical Handoff is feasible i.e. the handoff takes place if and only if RSS of the BS or Access Point (AP) is above the threshold. There is a close relationshipbetween the RSS readings and the distance from the mobile terminal to its point of attachment. Majority of existing horizontal handover algorithms use RSS as the main decision criterion, and RSS is an important criterion for VHD algorithms as well.

\section{Volume 4 Issue 12, December 2015}




\section{International Journal of Science and Research (IJSR) \\ ISSN (Online): 2319-7064}

Index Copernicus Value (2013): 6.14 | Impact Factor (2014): 5.611

\section{Vertical Handoff Decision Making Algorithms}

The complexity of the handoff decision process has led to the proposal of a number of vertical handoff decision algorithms for wireless access networks. The overview and characterization of different VHDA is prescribed below

\section{A. Received Signal Strength (RSS) based VHDAs: \\ The first approach is based on the traditional strategies of using the RSS that may be combined with other parameters. The RSS based vertical handoff decision algorithms compare the RSS of the current point of attachment with the RSS of the available point of attachment to make handoff decisions.}

\section{B. Cost function-based VHDAs:}

A vertical handoff decision cost function is a measurement of the benefit obtained by handing off to a particular network. It is evaluated for each network that covers the service area of a user. It is derived from a number of parameters and it is efficient, flexible and has a low implementation complexity. The main advantage brought by this approach is theincreased in percentage of user-satisfied requests and the reduction in blocking probability. However, no mention was made on the way in which QoS parameters were normalized, or how the weights were assigned.

\section{Multiple Criteria VHDAs:}

Multiple criteria VHD algorithms make handoff decisions based on several handoff criteria such as signal strength, network coverage area, network security, and service cost. They combine the various criteria in order to select the most suitable access network for a new service request or handoff. They are efficient and flexible, and have a medium implementation complexity. The handoff decision problem is a typical multiple criteria decision making (MCDM) problem since the handoff decision problem involves selecting a suitable access network among a number of candidate access networks with respect to several criteria (attributes). The cost function based algorithm and the computational intelligence based algorithm are based on multiple criteria. These algorithms are the currently used approaches for combining many decision criteria for vertical handoff decision. The MCDM can be divided into multiple attribute decision making (MADM) that deals with the problem of choosing an alternative from a set of alternatives which are characterized in terms of their attributes, and multiple objective decision making (MODM) that consists of a set of conflicting goals which cannot be achieved Simultaneously.Multiple attribute decision making (MADM) refers to making preference decision over the available alternatives that are characterized by multiple (usually conflicting) attributes.

\section{Analysis}

Out of these, the traditional and function based methods considers only the minimum number of parameters. So its performance on throughput and others may decrease while more constraints were added. User centric approach considers the user related parameters and preferences. But in some situation the network conditions and constraints should also be considered.

\section{Issues to be Addressed}

Future wireless systems will be based on heterogeneous wireless access technology. In order to provide seamless services many challenging issues to be solved.

QoS Issues- Mobile terminals carrying real time and non-real time traffic should be serviced with guaranteed QoS. To provide best network service several parameters to be considered.

TCP Performance Issues - When switching from low bandwidth, high data rate network to high bandwidth, low data rate network TCP performance should be considered for congestion.

Security Issues - Because of the wide coverage area when the sensitive data is transmitted it should be transferred in secured manner.

\section{Conclusion}

This paper presents an introduction to mobility management. An classification of different types of handoffs are also explained and a comprehensive survey on Vertical handoff decision making parameter's and decision making algorithms that helps in selection of most suitable vertical handoff decision making algorithm for selecting best network in NGHWN as per the requirement of the system.

\section{References}

[1] Dhanaraj Cheelu, M. RajasekharaBabu, P. Venkata Krishna" A Study of Vertical Handoff Decision Strategies in Heterogeneous Wireless Networks" International Journal of Engineering and Technology (IJET) Jun-Jul 2013.

[2] Sunisa Kunark,Raungrang Suleethira"Algorithmic Vertical Hnadoff Decision and Merit Network Selection across Hetrogenous wireless network.WSEAS Transaction on Communication,Jan 2013.

[3] Shusmita A. Sharna and ManzurMurshed" Impact on Vertical Handoff Decision Algorithm by the Network Call Admission Control Policy in Heterogeneous Wireless Networks" 23rd International Symposium on Personal, Indoor and Mobile Radio Communications (PIMRC), IEEE2012.

[4] Sandra Brigit Johnson, SaranyaNath P and T.Velmurugan"An Optimized Algorithm for Vertical Handoff in Heterogeneous Wireless Networks "Conference on Information and Communication Technologies (ICT 2013), IEEE2013.

[5] V. Anantha Narayanan and A. Rajeswari" An Intelligent Vertical Handover Decision Algorithm For Wireless Heterogeneous Networks" American Journal of Applied Sciences 2014. 


\section{International Journal of Science and Research (IJSR) \\ ISSN (Online): 2319-7064}

Index Copernicus Value (2013): 6.14 | Impact Factor (2014): 5.611

[6] Safdar Rizvi, Asif Aziz, N.M. Saad" An Overview of Vertical Handoff Decision Policies for Next Generation Wireless Networks" IEEE 2010.

[7] Fei Shi, Keqiu Li, YanmingShen" Seamless handoff scheme in Wi-Fi and WiMAX heterogeneous networks" science direct-Future Generation Computer Systems 26 (2010) pp 1403_1408.

[8] Ben-Jye Chang, Member, IEEE, and Jun-Fu Chen" Cross-Layer-Based Adaptive Vertical Handoff With Predictive RSS in Heterogeneous Wireless Networks" IEEE TRANSACTIONS ON VEHICULAR TECHNOLOGY, VOL. 57, NO. 6, NOVEMBER 2008 